\title{
Switchable Assembly of Ultra Narrow CdS Nanowires and Nanorods
}

Somobrata Acharya ${ }^{1}$, Israel Patla ${ }^{2}$, Joseph Kost ${ }^{3}$, Shlomo Efrima ${ }^{2}$ and Yuval Golan ${ }^{1 *}$

${ }^{1}$ Department of Materials Engineering, ${ }^{2}$ Department of Chemistry and the Ilse Katz Center for Meso and Nanoscale Science and Technology, ${ }^{3}$ Department of Chemical Engineering, Ben-Gurion University, Beer Sheva, Israel 84105

RECEIVED DATE (automatically inserted by publisher); E-mail Address: ygolan@bgu.ac.il

\section{Supporting Information}
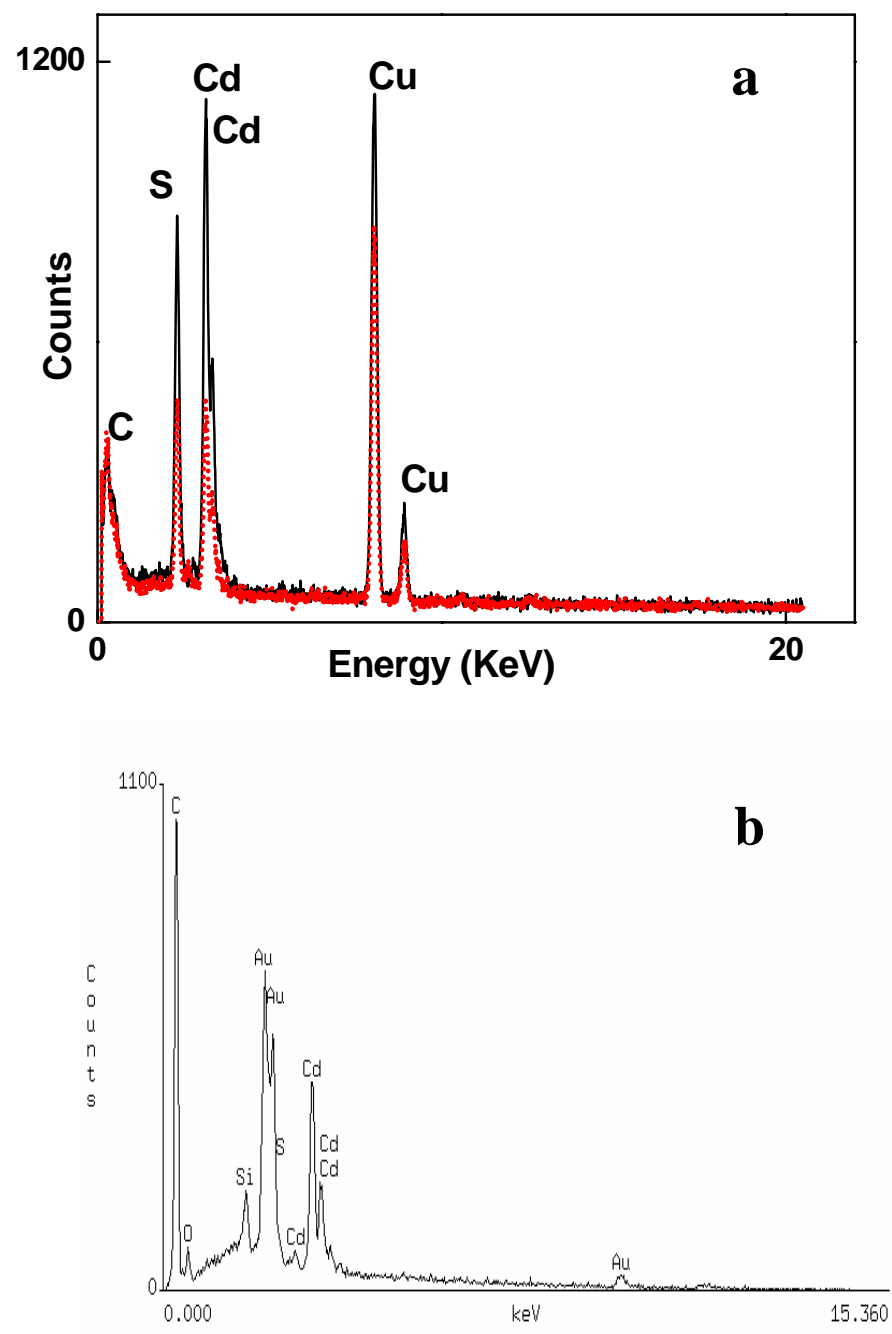


\begin{tabular}{|c|c|c|c|c|c|c|}
\hline \multirow{2}{*}{ Elements } & \multicolumn{2}{|c|}{ Wires } & \multicolumn{2}{c|}{ Rods } & \multicolumn{2}{c|}{ Microstrings } \\
\cline { 2 - 7 } & Cd & S & Cd & S & Cd & S \\
\hline Atom \% & 48 & 52 & 41 & 59 & 51 & 49 \\
\hline Weight \% & 76 & 24 & 70 & 30 & 78 & 22 \\
\hline
\end{tabular}

Figure S1. (a) EDS spectra taken in the TEM of CdS wires (black line) and rods (red dots) (b) EDS spectrum of microstrings measured in FEG-SEM on silicon wafer after gold sputtering. The table lists the atom and weight percentage of $\mathrm{Cd}$ and $\mathrm{S}$ in wires, rods and microstrings respectively.

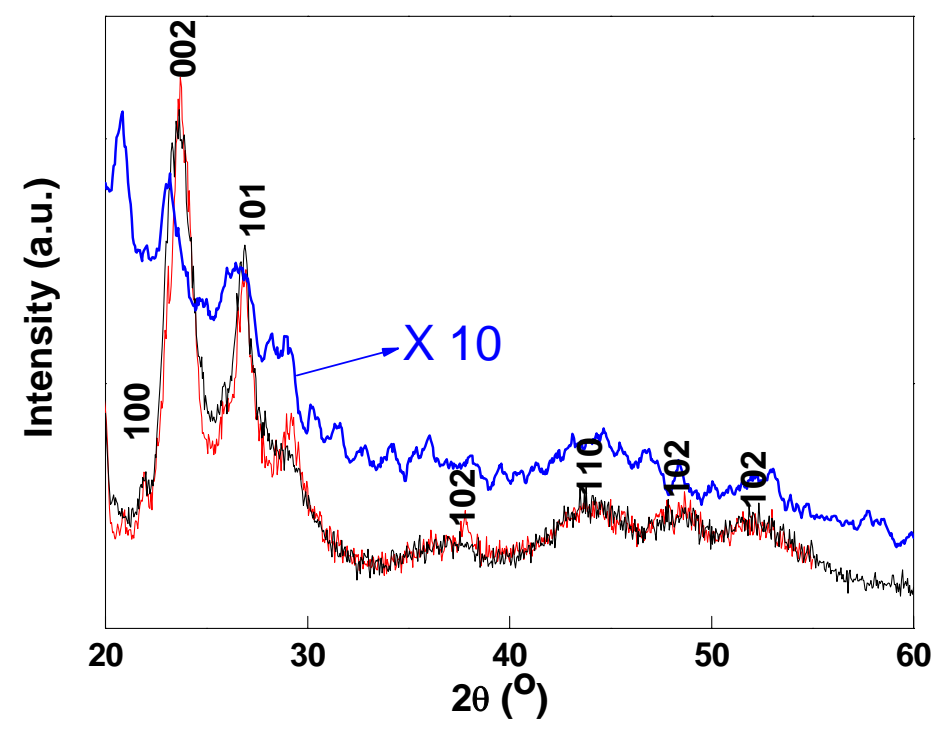

Figure S2. The XRD of powder samples of ordered wires (black line) and rods (red line) match well to the standard WZ structure of CdS (p6 $6_{3} \mathrm{mc}, \mathrm{a}=4.142 \AA, \mathrm{c}=6.724 \AA$; JCPDS 02-0549). The XRD pattern obtained from oriented CdS microstrings on Si support is shown in blue color. Since the WZ wires/rods are oriented with the $[00.1]_{\mathrm{WZ}}$ crystallographic axis parallel to the long axis of the wires/rods, the considerable decrease in intensity of the 00.2 peak in the diffraction signal obtained from the microstrings indicates that the $<00.1>$ crystallographic direction is now aligned parallel to the substrate. The peak at $2 \theta=29^{\circ}$ remains unidentified. However, it can not be attributed to the unique 200 reflection of zinc blende (cubic) CdS. 

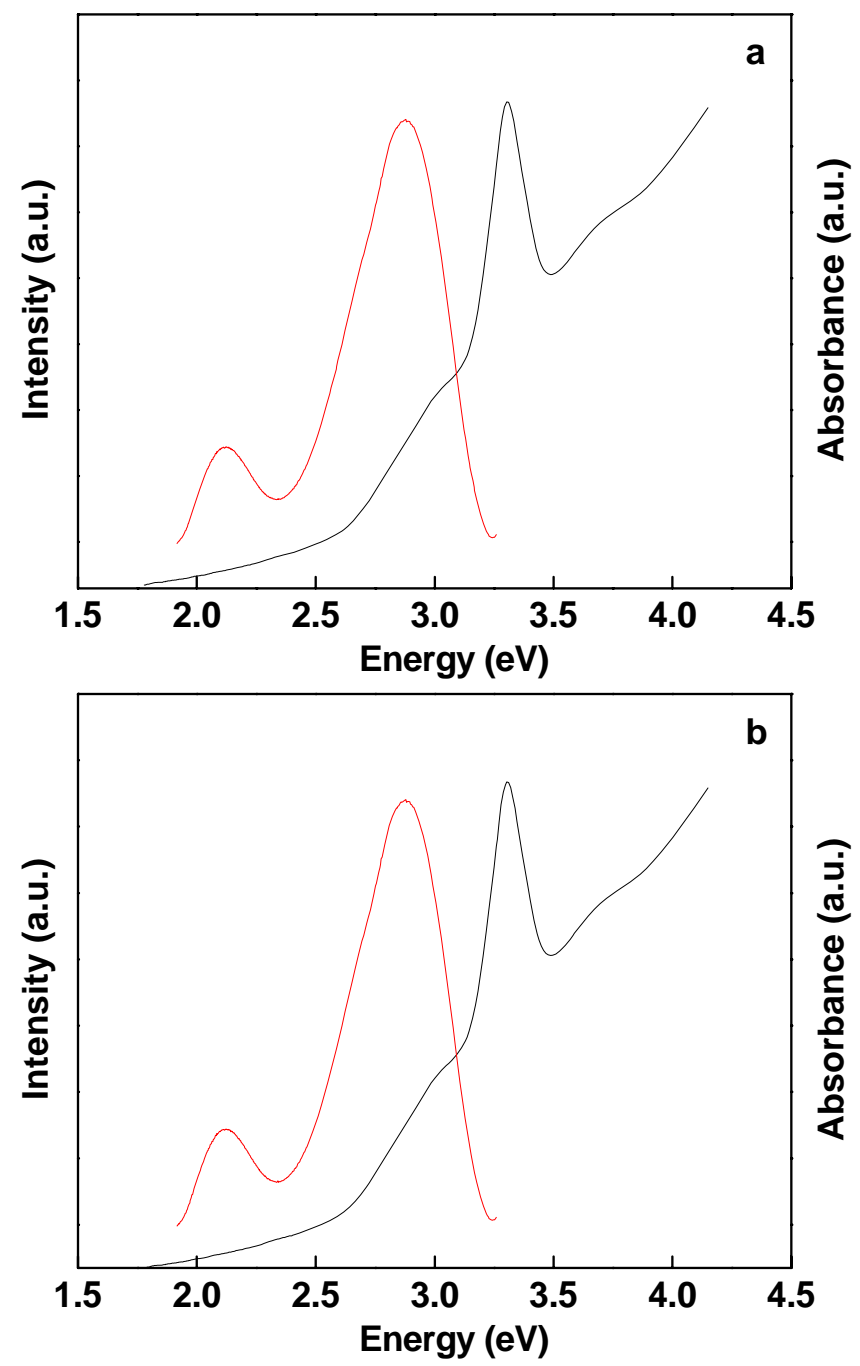

Figure S3. The room temperature PL (red curve) and UV-vis absorption (black curve) spectra of CdS nanowires (a) and nanorods (b) suspension in toluene. The absorption spectrum shows a sharp discrete absorbance feature $(3.3 \mathrm{eV})$ that is blue shifted compared to the bulk $(2.5 \mathrm{eV})$. The nanowires/rods exhibit a single strong band edge PL at $2.87 \mathrm{eV}$ with a weak shoulder shifted to lower energy at $2.1 \mathrm{eV}$. This low energy peak likely originates from surface or defect state. The sharp features in optical spectra are likely result from the very strong quantum confinement effect and narrow width distribution (the major controlling dimension). Interestingly, the nanorods show an 
identical spectrum as the wires in spite of relatively larger width, but still comparable to the Bohr radius of CdS, supporting that the width is the major controlling dimension.
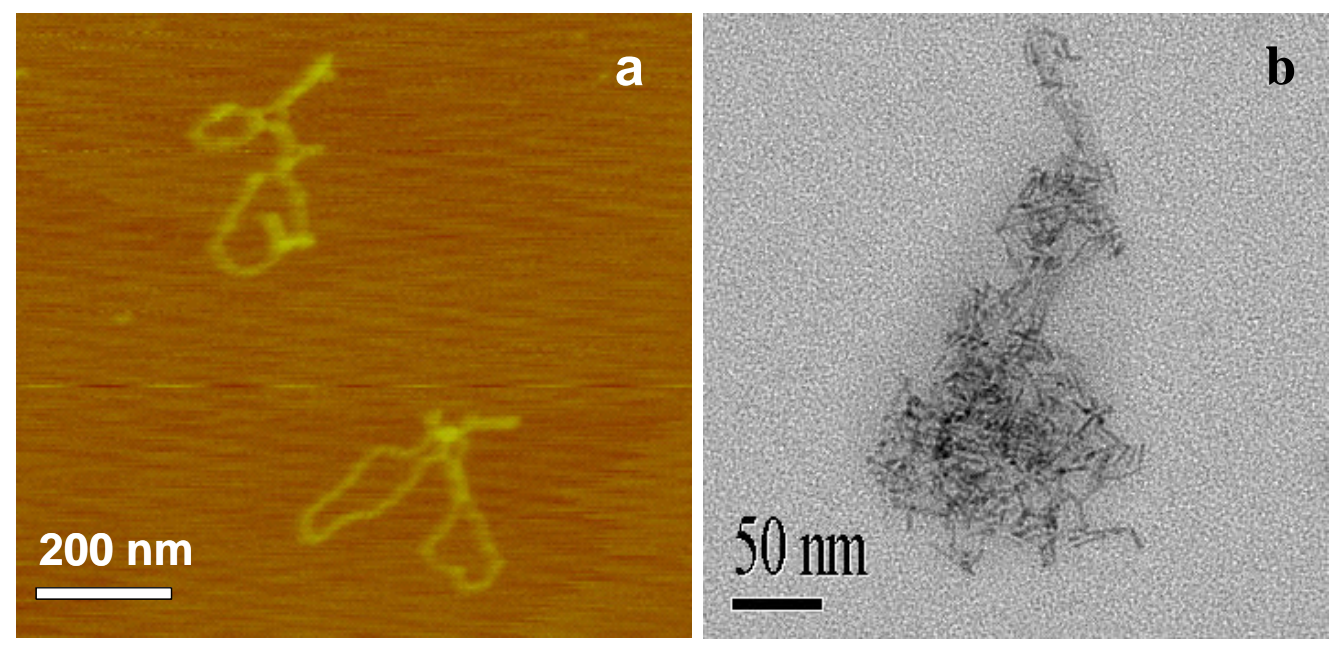

Figure S4. The assembly of mercaptoethylamine stabilized water soluble rods and plasmid DNA is demonstrated for their possible application in bio-nanotechnology. (a) AFM image of bare DNA molecules (The pCEFL plasmid DNA encoding for green fluorescence protein (GFP) was amplified in E-coli and purified by a Jetstar plasmid ${ }^{\circledR}$ Maxi kit) deposited on mica from water subphase demonstrating that length of the DNA molecules are typically $300 \mathrm{~nm}$. For AFM image the pCEFL plasmid DNA was diluted to $0.5 \mathrm{ngm} / \mu \mathrm{L}$ in a buffer containing $10 \mathrm{mM}$ HEPES (N-[2-Hydroxyethyl]piperazine-N'[2-ethanesulfonic acid], Sigma, 99.5\%), $\mathrm{pH} 7$, and $1 \mathrm{mM}$ concentration of $\mathrm{NiCl}_{2}, 6 \mathrm{H}_{2} \mathrm{O}$ (Fluka, $>98 \%$ ) (b) TEM of water soluble rods and DNA complex. Since DNA is transparent to the electron beam, the assembly profile is essentially a contribution from the nanorods bound to the DNA chains. The length and shape of the assembly is consistent with the AFM image. The end amine groups of the mercaptoethylamine stabilized rods in $\mathrm{pH}$ range of 5-6 are positively charged in aqueous phase. Such assembly can be expected due to the electrostatic interaction between cationic nanorods and negatively charged DNA phosphate groups. 

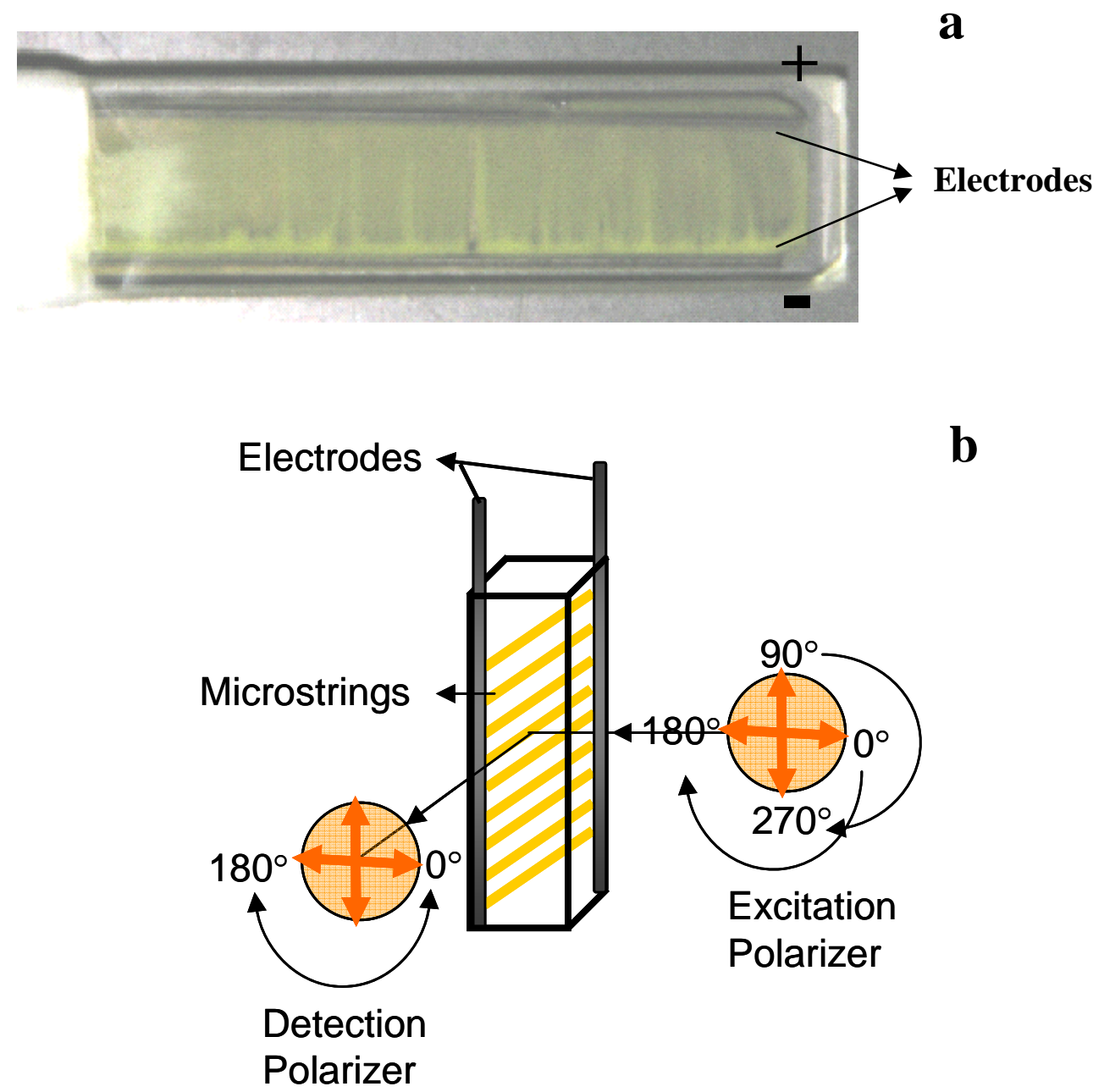

b

C

Electrodes

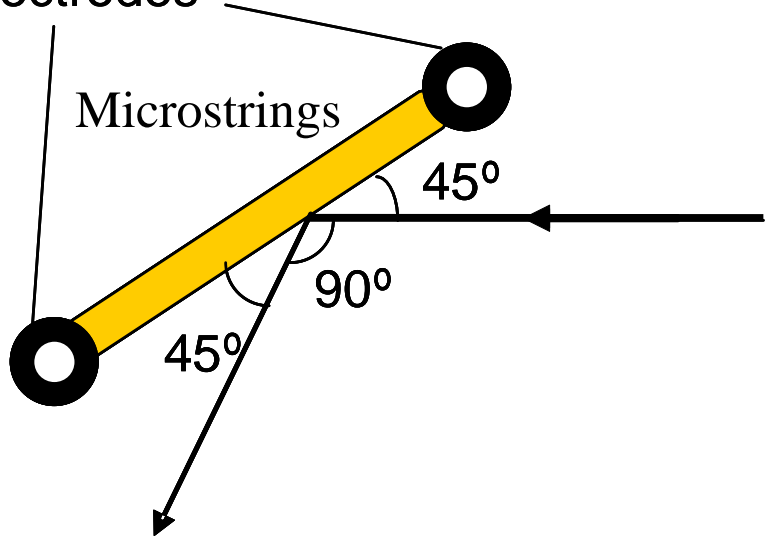


Figure S5. (a) A photograph of long range ordering of CdS nanowires into microstrings within a centimeter wide cuvett containing two graphite electrodes. The microstrings extend between two electrodes over the entire cuvett of $1 \mathrm{~cm}$. The strings are obtained after applying $500 \mathrm{~V}$ for $10 \mathrm{~min}$. The thick visible yellow lines are the bigger bundles (aggregates) of microstrings (b) A side view scheme of polarization measurement. We used a fluorometer (Fluorolog, Jobin Yvon) with excitation and detection polarizers. Two sets of measurements are performed to extract polarization ratio (r). In the first set, the excitation polarizer is rotated form $0^{\circ}$ to $180^{\circ}$ (from parallel to parallel setting with the microstrings), in the second set, the excitation polarizer is rotated form $90^{\circ}$ to $270^{\circ}$ (from perpendicular to perpendicular setting with the microstrings). In each measurement, the detection is done from $0^{\circ}$ to $180^{\circ}$ (parallel emission) (c) A top view of the experimental set-up for polarization measurements. 